Volume 10, No.2, March - April 2021

International Journal of Advanced Trends in Computer Science and Engineering

Available Online at http://www.warse.org/IJATCSE/static/pdf/file/ijatcse741022021.pdf

https://doi.org/10.30534/ijatcse/2021/741022021

\title{
Dynamic 5G Network Slicing
}

\author{
Joshi Vilas Ramrao ${ }^{1}$, Dr.Amit Jain ${ }^{2}$ \\ ${ }^{1,2}$ Department Electronics Communication Engineering, Electronics Communication Engineering \\ OPJS University, Churu, Rajasthan, India
}

\begin{abstract}
5G network slicing is the use of network virtualization to divide single network connections into multiple distinct virtual connections that provide different amounts of resources to different types of traffic. A 5G NS (Network Slicing) instance is composed of a set of virtual network function (VNF) instances to form the end-to-end (E2E) virtual network for the slice to operate independently. The deployment of a NS is a typical virtual network embedding (VNE) problem. The proposed algorithm consists of three parts. First, we devise a Holt-Winters (HW) prediction algorithm to determine traffic demand for network slices. This method is intended to avoid frequent changes in network topology. Second, we propose a virtual network function (VNF) adaptive scaling strategy to reasonably determine the number of VNFs and resources required for network slices to avoid resource wastage. Finally, we develop a proactive online algorithm to deploy network slices.
\end{abstract}

Key words: 5G network, slicing, virtual network function VNF

\section{INTRODUCTION}

Network slicing is a type of virtual networking architecture in the same family as software-defined networking (SDN) and network functions virtualization (NFV) - two closely related network virtualization technologies that are moving modern networks toward software-based automation. SDN and NFV allow far better network flexibility through the partitioning of network architectures into virtual elements [1]. In essence, network slicing allows the creation of multiple virtual networks atop a shared physical infrastructure. 5G uses software-defined networking and network functions virtualization for the partitioning of network architectures into virtual elements [2]. 5G Network slicing allows network operators to have a portion of the network provide the exact features a segment of the customer base needs. GSMA Intelligence hypothesizes that $5 \mathrm{G}$ network architectures will need network slicing in order to adapt and provide the specific needs of unique industries [3].

Scholars have recognized $5 \mathrm{G}$ networks as crucial in enabling network operators to enter the vertical industry market. Dense and crowded environments, such as stadiums and subway stations, exemplify the disadvantages of excessive demand and traffic from the user side. Network slicing can effectively address this problem. Specifically, network slicing technology can divide a physical network into multiple virtual network slices according to network slice providers' needs, thereby improving network resource utilization, reducing network operators' costs and energy consumption, and enhancing network users' experience quality. Network function virtualization (NFV) and software-defined networking (SDN) are key technologies for constructing 5G network slices [4]. Network slicing technology divides the network into fine-grained network functions (NFs) and deploys them on the virtual platform through containers. Network slices are then further configured and managed through SDN and NFV to provide users an efficient, programmable, and scalable network service. While network slicing technology can ensure greater performance and more efficient networks, it also brings new obstacles: network slice deployment is a major challenge in network slicing technology[5].

A key issue in deploying network slices is how to reduce the costs and energy consumption of network operators while ensuring network slice service quality. Current network slicing is plagued by several difficulties. First, most relevant research has focused on static deployment of network slices; in reality, however, the request process of network slices is dynamic, the slice user distribution is uneven and static resource allocation leads to wasted resources and costs. Second, VNF deployment in network slices affects slices' VNF routing path, thus influencing the delay quality of these slices. Third, the costs associated with network operators and network energy consumption cannot be neglected [6]. To address these obstacles, 
this paper investigates the dynamic deployment of network slices, establishes a network slicing management and orchestration architecture, and designs a $5 \mathrm{G}$ network-oriented prediction-assisted adaptive network slice expansion algorithm to dynamically create and deploy network slices. In our algorithm, we first consider a dynamic system in which a network slice request is dynamically generated [7]. We then propose a HW prediction algorithm to predict the traffic rate of network slices. This algorithm can minimize prediction errors and simultaneously reduce the cost and delay of creating VNFs in network slices [8]. Next, we construct a VNF adaptive scaling strategy to determine the number of VNFs and resources; this strategy can conserve network resources by (a) setting VNFs reasonably and (b) reducing the number of VNFs. We then discuss network operators' costs and energy consumption and construct a joint optimization problem. Finally, we propose a proactive online network slice deployment algorithm that can dynamically deploy network slices while meeting delay requirements to minimize network costs and energy consumption [10].

\section{LITERATURE SURVEY}

In [1], Chengli Mei et al investigated the 5G E2E NS deployment problem with sharable VNF instances. We formulate the multiple coupled NS virtual network embedding problem through an ILP formulation. Our design goal is to minimize the resource consumption by integrating sharable VNF instances. A VNF-sharing based NSR deployment algorithm is designed. The NSRs acceptance ratio, instantiated number of VNFs and CPU consumption under different physical network scales, NSRs resource requirements and sharable resources percentage is investigated.

In [2], Zhou et al considered the dynamic nature of a network when investigating network slice deployment. First, the network slice management and orchestration architecture is built. Then a joint optimization of cost and energy consumption is constructed. Network slice service requests changed dynamically over time and demonstrated certain trends and periodicity. A prediction algorithm to forecast the network slice traffic rate to avoid frequent updates to network topology is proposed. Then used the VNF adaptive scaling strategy based on our prediction results to determine the number of VNFs and resources to conserve resources and reduce network slice deployment costs. In addition, a proactive online algorithm to deploy network slices and elaborated on the VDA and LRA for VNF instance configuration and link routing is also proposed.

In [3], novel software data path architecture that enables such network slicing with assured QoS in $5 \mathrm{G}$ multi-tenant networks is designed and implemented. Highly flexible and customisable definition of network slicing is also allowed to be aligned with different existing definitions on demand and at run time. The proposed architecture has been prototyped based on the popular Open Virtual Switch (OVS), and empirically validated to demonstrate the deployment and management of network slices with the above capabilities. Intensive scalability results are provided where more than 8,192 network slices are achieved simultaneously with warranted QoS through performance isolation in terms of bandwidth and delay in a real softwarised $5 \mathrm{G}$ multi-tenant infrastructure at speeds of up to $10 \mathrm{Gbps}$.

In [4], a markerless human-robot interface that incorporates Kalman filters (KFs) and particle filters (PFs) to track the posture of human hands is proposed. This method allows one operator to control dual robot manipulators by using his/her double hands without any contact devices or markers. The algorithm employs Leap Motion to determine the orientation and the position of the human hands. Although the position and the orientation of the hands can be obtained from the sensor, the measurement errors increase over time due to the noise of the devices and the tracking error. The PFs and KFs are used to estimate the position and the orientation of the human hand. Due to the limitations of the perception and the motor, a human operator cannot accomplish high-precision manipulation without any assistance. An adaptive multispace transformation is employed to assist the operator to improve the accuracy and reliability in determining the posture of the manipulator. The greatest advantage of this method is that the posture of the human hands can be estimated accurately and steadily without any assistant markers. The human-manipulator interface system was experimentally verified in a laboratory, and the results indicate that such an interface can successfully control dual robot manipulators even if the operator is not an expert.

In [5], a fundamental problem for the slicing of $5 \mathrm{G}$ transport networks, i.e., reliable $\mathrm{VN}$ embedding with dedicated protection in an EON is addressed. To reduce resource overbuild of dedicated protection, we exploit bandwidth squeezing and VLink demand splitting over multiple SPaths, while leveraging the flexibility offered by an EON. This splitting model not only provides the opportunity to split a VLink demand across multiple SPaths but also across multiple spectrum segments of a SPath. 
The opportunity to have multiple splits over the same path allows to save additional spectrum compared to a model that does not allow splitting on the same path, and proposed heuristic performs close to the optimal solution. For instance, multiple splits enable us to guarantee a BSR $40 \%$ with only $10 \%$ additional spectrum resources. This translates into similar VN blocking ratio up to $40 \%$ BSR. Our discrete event simulation also shows that similar spectrum occupancy is achieved on EON links irrespective of BSR requirement for a given $\mathrm{VN}$ arrival rate. However, spectrum occupancy increases with the increase in $\mathrm{VN}$ arrival rate.

In [6], the problem of allocating resources to demands of different priority slices in a multi-slice network for both offline and online modes is discussed. This was based on the proposed SKM strategy for the purpose of effectively allocating available resources to serve demands in physical network paths. Since the computational load to find paths from the source node to destination node and their selection for the demands is huge even when using our proposed algorithm, then, the algorithm is forwarded to NFV architecture in order to provide the huge computational capacity required for the network service. SKM can be adapted to allocate bandwidth resources and any general resource management where resources require a reservation in addition to allocation stages, between different entities such as NFV service chain allocation and, of course, network slicing in future networks. Simulation results showed that thanks to our proposed algorithm, not only can we significantly improve the overall network usage, but also achieve the appropriate QoS and prioritized admission control for different E2E slice users. Moreover, our proposed algorithm can accept demands of considerable size, hence, guaranteeing a high admission of higher priority slices compared to other efficient schemes. This is mainly because the proposed algorithm implements a policy for resources selection that tends to increase the resources usage efficiency. Besides, it was proven that the algorithm is scalable with increasing substrate network demand sizes.

In [7], Li, Taihui et al proposed the DQN based autonomous resources allocation framework for the next generation mobile networks. The proposed scheme takes into account the influence of E2E to ensure the maximum access rate of the whole system. In this system, author considers rate constrained slices and delay constrained slices. Different slices have different constraints and resource requirements. Reasonable resource allocation and dynamic adjustment between slices make the system access more users. DQN was used by the slices to adjust resources. The reward of DQN is the E2E access rate, which was solved by break the P1 into two sub-problems. Different algorithms are designed to solve sub-problem so that we can get the reward of DQN and autonomously adjust resource. The simulation results show that DQN can dynamically change the resource allocation according to the system access rate in static or dynamic environment. The system access rate can be higher than $98 \%$ in static environment which is the best compared with ASO and BSC schemes. When users moves below $60 \mathrm{~m} / \mathrm{min}$, the average access rate of DQN scheme is higher than $97 \%$. Compared with ASO schemes, the average access rate is increased by $9 \%$ for delay constrained slices and $5 \%$ for rate constrained slices.

In [8], Jie Mei proposed a self-sustained RAN slicing framework, which integrates the self-management of network resources with multiple granularities, the self-optimization of slicing control performance, and self-learning together to achieve an adaptive control strategy under unforeseen network conditions. The proposed RAN slicing framework is hierarchically structured, which decomposes the RAN slicing control into three levels, i.e., network-level slicing, next generation NodeB (gNodeB)-level slicing, and packet scheduling level slicing. At the network level, network resources are assigned to each gNodeB at a large timescale with coarse resource granularity. At the gNodeB-level, each gNodeB adjusts the configuration of each slice in the cell at the large timescale. At the packet scheduling level, each gNodeB allocates radio resource allocation among users in each network slice at a small timescale. Furthermore, we utilize the transfer learning approach to enable the transition from a model-based control to an autonomic and self-learning RAN slicing control. With the proposed RAN slicing framework, the QoS performance of emerging services is expected to be dramatically enhanced.

In [9], a data-driven multiobjective optimization framework for hyperdense $5 \mathrm{G}$ network planning with practical case studies used to illustrate added value compared to contemporary network planning and optimization approaches is proposed. Comparative results from the case study with real network data reveal potential performance and cost improvements of hyperdense optimized networks produced by the proposed framework due to increased use of contextual data of planning area and focus on objectives that target demand satisfaction.

In [10], based on the Performance Evaluation Process Algebra (PEPA), a method is proposed aiming at enabling the systematic analysis of the performance and scalability of network services in the $5 \mathrm{G}$ core. The former corresponds to the session establishment in a $5 \mathrm{G}$ network slice. The latter 
corresponds to the user registration process in a network slice for Vehicle-to-Everything. Results show the usefulness of our method to model $5 \mathrm{G}$ core network services and dimension the capacity of slices that implement them. Furthermore, the validation results corroborate, in terms of accuracy, that our PEPA-based method measures performance metrics (throughput, average response time, and processor utilization) with negligible difference regarding a traditional approach like the Layered Queuing Network model.

\section{PROPOSED SYSTEM}

The network slice management and orchestration (MO) architecture is illustrated in Fig. 1. This architecture supports dynamic deployment of $5 \mathrm{G}$ network slices on top of the ETSI NFV architecture [29]. The architecture includes five main modules: the communication service management function (CSMF), network slice descriptor (NSD), network slice management function (NSMF), network slice subnet management function (NSSMF), and cloud network.

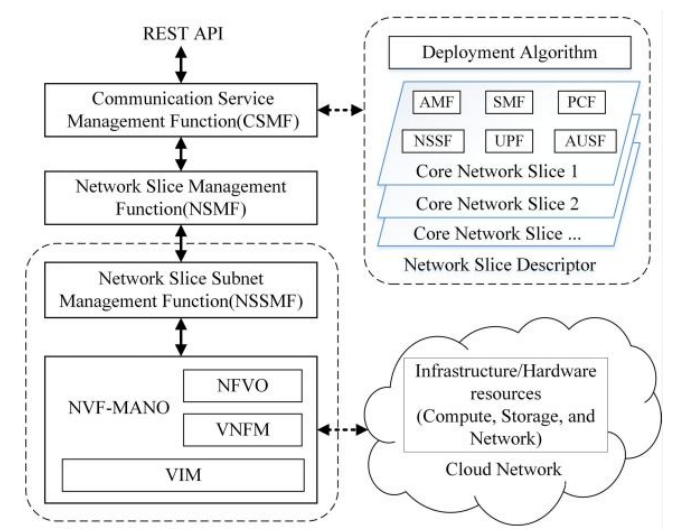

Fig 1 the network slice MO architecture

Network slices are deployed as follows. In the first step, the network operator uses the northbound interface (e.g., REST API) to transmit a network service request to the CSMF module. In the second step, the CSMF transforms the network service request into a network slice request, determines the deployment positions of network slices using the proactive online algorithm in the NSD module, and transmits it to NSMF. In the third step, NSMF transforms the need for network slices into the need for separate subnets and then coordinates and manages network slices in separate subnets via NSSMF. The network-based resource allocation depends on NSSMF with NFV management and orchestration (NFVMANO) [30]. NFV-MANO is responsible for running the algorithm proposed in this paper to determine the number of VNFs in network slices along with optimal deployment locations. NFV-MANO is mainly composed of NFV orchestration (NFVO) along with a VNF manager and virtualized infrastructure manager. Finally, network operators allocate resources from the cloud network to deploy network slices.

\section{CONCLUSION}

Network slicing is a key technology in fifth-generation (5G) mobile networks. Slicing divides a physical network into multiple dedicated logical networks to meet the requirements of diverse use cases. Efficient slice deployment algorithms are critical in reducing network operators' costs and energy consumption and in providing users better service. Many researchers have focused on static deployment when investigating network slices, effectively ignoring network operators' requirements for the dynamic deployment and expansion of such slices. In this paper, we first construct a joint optimization problem of cost and energy consumption. Then, we propose a prediction-assisted adaptive network slice expansion algorithm to deploy network slices dynamically.

\section{REFERENCES}

1. Chengli Mei, Jiayi Liu, Jinyan Li, Lei Zhang, and Menghan Shao, "5G Network Slices Embedding with Sharable Virtual Network Functions", JOURNAL OF COMMUNICATIONS AND NETWORKS, VOL. 22, NO. 5, OCTOBER 2020

2. Zhou, Jinhe; Zhao, Wenjun; Chen, Shuo (2020). Dynamic Network Slice Scaling Assisted by Prediction in 5G Network. IEEE Access, 8() , 133700-133712. doi:10.1109/access.2020.301 0623

3. Escolar, Antonio Matencio; Wang, Qi; Calero, Jose M Alcaraz (2020). SliceNetVSwitch: Definition, Design and Implementation of $5 \mathrm{G}$ Multi-tenant Network Slicing in Software Data Paths. IEEE Transactions on Network and Service Management, (), 1-1. doi:10.1109/tnsm.2020.3029653

4. Yu, Hao; Musumeci, Francesco; Zhang, Jiawei; Tornatore, Massimo; Ji, Yuefeng (2020). Isolation-Aware 5G RAN Slice Mapping over WDM Metro-Aggregation Networks. Journal of Lightwave Technology, (), 1-1. doi:10.1109/JLT.2020.2973311

5. Shahriar, Nashid; Taeb, Sepehr; Chowdhury, Shihabur Rahman; Zulfiqar, Mubeen; Tornatore, Massimo; Boutaba, Raouf; Mitra, Jeebak; Hemmati, Mahdi (2020). Reliable Slicing of $5 \mathrm{G}$ Transport Networks with Bandwidth Squeezing and Multi-path 
Provisioning. IEEE Transactions on Network and Service Management, (), 1-1. doi:10.1109/TNSM.2020.2992442

6. Ahmed El-Mekkawi; Xavier Hesselbach; Jose Ramon Piney, "Novel NFV Aware Network Service for Intelligent Network Slicing Based on Squatting-Kicking Model”, 10.1109/ACCESS.2020.3044951

7. Li, Taihui; Zhu, Xiaorong; Liu, Xu (2020). An End-to-End Network Slicing Algorithm Based on Deep Q-Learning for 5G Network. IEEE Access, 122229-122240. doi:10.1109/ACCESS.2020. 3006502

8. Jie Mei; Xianbin Wang; Kan Zheng, “An intelligent self-sustained RAN slicing framework for diverse service provisioning in 5G-beyond and 6G networks", Intelligent and Converged Networks ( Volume: 1, Issue: 3, Dec. 2020), DOI: 10.23919/ICN.2020.0019

9. Haile, Beneyam B.; Mutafungwa, Edward; Hamalainen, Jyri (2020). A Data-Driven Multiobjective Optimization Framework for Hyperdense 5G Network Planning. IEEE Access, 1-1. doi:10.1109/ACCESS.2020.3023452

10. Arteaga, Carlos Hernan Tobar; Ordonez, Armando; Rendon, Oscar Mauricio Caicedo (2020). Scalability and Performance Analysis in $5 \mathrm{G}$ Core Network Slicing. IEEE Access, 8(), 142086-142100. doi:10.1109/ACCESS.2020. 301359 